\title{
KIF18A is a potential prognostic factor and promotes tumor progression in oral tongue squamous cell carcinoma
}

\author{
XIAOFeI LV ${ }^{1, *}$; XI YU ${ }^{2, *}$; Jie XU ${ }^{3}$; MingYI WANG ${ }^{4}$; Cheng PENG ${ }^{1, *}$ \\ 1 Department of Stomatology, the Second Hospital of Tianjin Medical University, Tianjin, 300211, China \\ 2 Department of Anesthesiology, the Second Hospital of Tianjin Medical University, Tianjin, 300211, China \\ 3 Key Laboratory of Cancer Prevention and Therapy, National Clinical Research Center for Cancer, Tianjin Medical University Cancer Institute and Hospital, \\ Tianjin, 300060, China \\ 4 Department of Oral \& Maxillofacial-Head \& Neck Oncology, Shanghai Ninth People's Hospital, College of Stomatology, Shanghai Jiaotong University; \\ Shanghai Key Laboratory of Stomatology \& Shanghai Research Institute of Stomatology, National Clinical Research Center of Stomatology, Shanghai, \\ 200011, China
}

Key words: KIF18A, Tongue cancer, Squamous cell carcinoma, Progression

\begin{abstract}
The kinesin family member 18A protein was dysregulated in several human cancers and involved in cancer progression. However, the significance in oral tongue squamous cell carcinoma (OTSCC) has not been studied. The present study was intended to explore the functions of KIF18A in oral tongue squamous cell carcinoma. The immunohistochemistry (IHC) assay was performed to assess the relationships between the KIF18A protein expression level and clinical-pathological features of the patients. The biological functions of KIF18A in OTSCC cells were investigated by the experiments in vitro and in vivo. Based on immunohistochemistry, we found that KIF18A was correlated with the clinical-pathological features of OTSCC patients. High expression of KIF18A was associated with the lymph node metastasis, and clinical stages. In vitro experiments revealed that silencing of KIF18A significantly inhibited the expression of the proliferation and migration related proteins such as Ki67, proliferating cell nuclear antigen, matrix metalloproteinase-7 and matrix metalloproteinase-9, and thereby inhibiting the proliferation, migration and invasion of tumor cells. In vivo, knocking-down of KIF18A could inhibit the tumor growth in nude mice. In conclusion, we found KIF18A promoted tumor progression in vivo and in vitro and might become an effective target for the treatment of OTSCC.
\end{abstract}

\section{Introduction}

Oral tongue squamous cell carcinoma (OTSCC) is the most common type of oral cancer in the world (Ferlay et al., 2015). The median age of diagnosis is 62-year-old; the incidence of young adults is increasing recently, particularly those under 45-year-old (Li et al., 2013; Patel et al., 2011). The high incidence rate may be associated with the increase in the consumption of alcohol and tobacco products in this population (Anaya-Saavedra et al., 2008; Elango et al., 2007; Pintos et al., 2008). OTSCC is highly invasive and characterized by early metastasis and poor prognosis (Chiodo et al., 1986; Sciubba, 2001). Though the improvement of the treatment approaches and molecular

\footnotetext{
*Address correspondence to: Cheng Peng, peng_cheng2013@126.com

${ }^{\#}$ These authors have contributed equally to this work

Received: 09 July 2021; Accepted: 26 July 2021
}

mechanisms of OTSCC, effective target treatment for OTSCC remains needed (Ribeiro et al., 2016; Arteaga and Baselga, 2012). The effective target treatment for OTSCC is urgently needed to improve the prognosis.

As a member of microtubule-associated kinesin superfamily (KIF), KIF18A has been shown to be involved in the progression of multiple types of cancers (Braun et al., 2015; Chen et al., 2016; Kasahara et al., 2016). Kinesins play pivotal roles in regulating cellular processes, such as cell shape, cytoskeleton dynamics, intracellular organelle/macromolecule transportation and cell division, therefore affecting carcinogenesis (Stumpff et al., 2008). KIF18A plays a key role in regulating chromosome aggregation and inhibiting centromere movement (Zhu, 2005; Mayr et al., 2007). Dysregulation of KIF18A may lead to chromosomal instability. Previous studies showed that KIF18A was overexpressed in several types of cancers including renal cancer, breast cancer, and hepatocellular carcinoma (HCC) (Luo et al., 2018; Zhang et al., 2010; Frisch and Screaton, 2001). 
In breast cancer, functional analysis of KIF18A showed that KIF18A regulated cell proliferation and cell migration through the phosphatidylinositol 3-kinase (PI3K)-AKT signaling pathway and modulation of microtubule dynamics (Zhang et al., 2010). Also, knockdown of KIF18A dramatically induced cell anoikis in human breast cancer cells (Frisch and Screaton, 2001). Another study revealed that via the cell cycle signaling pathway and MMP-7/MMP9-related signaling pathway, KIF18A was involved in proliferation, invasion, and metastasis of hepatocellular carcinoma cells (Luo et al., 2018). Given the critical features of KIF18A, we further investigated its function in OTSCC.

In this study, we demonstrated that KIF18A was significantly overexpressed in OTSCC tissues and the high KIF18A expression was significantly associated with lymph node metastasis and clinical stages (Phases I-II, III-IV) of OTSCC patients. Overexpressed KIF18A was associated with tumor cell proliferation, migration and invasion in OTSCC in vivo and in vitro. Thus, our findings indicated that KIF18A might serve as a potential target for OTSCC patients.

\section{Materials and Methods}

Patients and samples

82 patients diagnosed with OTSCC in clinically and pathologically standards in the second hospital of Tianjin medical university and Shanghai Ninth People's Hospital were included in this study between the year 2010 and 2018. Written informed consents were obtained from all patients before the first surgical treatment. Patients' clinical and pathological data including age, gender, tumor stage, differentiation, clinical stage and lymph metastasis were analyzed. The histopathology of each specimen was reconfirmed at the Second Hospital of Tianjin Medical University.

\section{Immunohistochemistry staining}

Tumor specimens of OTSCC patients were fixed with $10 \% \mathrm{v} / \mathrm{v}$ formalin solution, embedded in paraffin, then cut into $5-\mu \mathrm{m}$ sections and baked at $70^{\circ} \mathrm{C}$ for $45 \mathrm{~min}$. Immunohistochemistry two-step test kit (Biotin-Streptavidin HRP Detection Systems, Beijing ZSGB-BIO Technologies, Co.) was used based on manufacturer's guidelines and recommendations. Then, the paraffin sections were placed into a microwave oven for $15 \mathrm{~min}$ to repair the antigens and were then cooled at room temperature. Endogenous peroxidase was blocked, and sections were incubated by using a KIF18A Polyclonal Antibody (PA558728, Thermo Fisher Scientific; 1:250 dilution) overnight at $4^{\circ} \mathrm{C}$, followed by a goat anti-rabbit secondary antibody at $37^{\circ} \mathrm{C}$ for $1 \mathrm{~h}$. Sections were stained with 3,3-diaminobenzidin (DAB) at room temperature for $10 \mathrm{~min}$ and images were then recorded by microscopy (Olympus BX43). All samples were evaluated by two independent pathologists. We used an evaluation system that combining the proportion and intensity of tumor cells to assess the staining results. The rate of positively stained cells was scored on a 3-point scale (less than $5 \%$ positive tumor cells were $0,5-50 \%$ positive tumor cells were 1 , and more than $50 \%$ positive tumor cells were 2$)$. The staining intensity was classified on a 3 -point scale ( 0 means no staining or light staining, 1 means intermediate staining,
2 means dark staining). Based on the sum of the staining intensity score and the percentage score, we defined the KIF18A expression level: 0-2 for KIF18A low expression and 3-4 for high expression. Without knowledge of pathological grade and clinical data, two experienced pathologists independently observed staining levels.

\section{Cell culture}

Human OTSCC cell lines (CAL27 and TCA8113) were purchased from the Beijing Type Culture Collection. According to the instructions, CAL27 cell was cultured in DMEM medium containing $1 \%$ penicillin-streptomycin and $10 \%$ fetal bovine serum (FBS, Gibco, Grand Island, NY, USA). TCA8113 cell line was cultured RPMI-1640 medium containing $1 \%$ penicillin-streptomycin and 20\% FBS. Both cell lines were maintained at $37^{\circ} \mathrm{C}$ with $5 \% \mathrm{CO}_{2}$.

\section{Transfection and generation of stable cell lines}

Short hairpin RNA oligonucleotide sequence (shRNA) targeting KIF18A was used to decrease its expression was AAAGCAGCTGGATTTCATAAAGT and a scramble sequence was used as a control. Plasmids were purchased from Vigene (Cat\# SH816146, Vigene Biosciences, Rockville, USA). The shRNA plasmids were transfected into cancer cells by the use of Invitrogen Lipofectamine ${ }^{\circledR} 3000$ (Thermo Fisher Scientific, Inc., USA). 100,000 cell per well in six-well plates according to the manufacturer's protocol, 3 groups were set, including: sh-KIF18A group, negative Control group (transfected with scrambled sequence). After 48-h transfection, cells were collected, followed by RT-qPCR and immunoblotting to verify the silencing efficiency. Then the KIF18A stable depleted-cell lines was screened and used for the in vitro and in vivo assays.

\section{$R T-q P C R$}

The total RNA was extracted at $2 \mu \mathrm{g}$ was reversely transcribed to cDNA template by the M-MuLV First Strand cDNA Synthesis Kit (B532435, Sangon Biotech, China). RT-qPCR was performed on a Smart Cycler using SGExcel FastSYBR Mixture (with ROX) (B532954, Sangon Biotech, China). Primer sequences are listed as follow: KIF18A (Forward) 5'-TGCTGGGAAGACCCACACTAT-3', and (Reverse) 5'-GCTGGTGTAAAGTAAGTCCATGA-3'; GAPDH (Forward) 5'-CAAGGTCATCCATGACAACTTTG-3', and (Reverse) 5'-GTCCACCACCCTGTTGCTGTAG-3'. All experiments were performed three times.

\section{Immunoblotting}

Cells and xenograft tumor tissues were lysed by RIPA buffer (Beyotime, ShangHai, China), and protein concentrations were measured by using the bicinchoninic acid (BCA) Assay kit (Biosharp, Hefei, China). Lysates of equal protein concentrations were electrophoresed by $10 \%$ SDS-PAGE gel and transferred onto PVDF membrane (Thermo, American). Membranes were blocked by $5 \%$ non-fat milk for $1 \mathrm{~h}$ at room temperature and incubated with the primary antibodies at $4^{\circ} \mathrm{C}$ overnight. The primary antibodies used were as follow: anti-KIF18A (rabbit, 1:150 dilution, Thermo Fisher Scientific), anti- $\beta$-actin (mouse, 1:5000 dilution, bioPM), antiKi67 (rabbit, 1:500 dilution, Abcam), anti-PCNA (mouse, 
1:500 dilution, Thermo Fisher Scientific), anti-MMP7 (rabbit, 1:500 dilution; ABclonal), and anti-MMP9 (rabbit, 1:500 dilution, ABclonal). The membranes were incubated with the HRP-conjugated secondary antibody (polyclonal goat antirabbit/mouse, Cell Signaling Technology) for $1 \mathrm{~h}$ at room temperature and visualized by chemiluminescence.

\section{Colony formation assay}

CAL27 and TCA8113 cells were plated into 6-well plates with 500 cells per well and cultured at $37^{\circ} \mathrm{C}$ with $5 \% \mathrm{CO}_{2}$ for 2 weeks. Then, cells were fixated with $4 \%$ paraformaldehyde for $15 \mathrm{~min}$ and stained $10-30 \mathrm{~min}$ with Giemsa reagent. The individual colonies, with diameters $>2 \mathrm{~mm}$, were counted under a microscope. All experiments were performed three times.

\section{MTT assay}

$5 \times 10^{3} /$ well of CAL27 and TCA8113 cells were plated in 96-well plates and cultured at $37^{\circ} \mathrm{C}$ with $5 \% \mathrm{CO}_{2}$. Then, we used $10 \mu \mathrm{L}$ of $5 \mathrm{mg} / \mathrm{mL}$ MTT reagent to replace the cell culture medium of each well for $4 \mathrm{~h}$ at the indicated time. Dimethyl sulfoxide (DMSO, Sigma) was added into each well for half an hour. The absorbance was measured at $570 \mathrm{~nm}$ wavelength. All experiments were performed three times.

\section{Wound healing assay}

$4 \times 10^{5} /$ well of CAL27 and TCA8113 cells were plated in 6-well plates. After the cells reached 95\% confluency, a sterile $100 \mu \mathrm{L}$ pipette tip was used to create a scratch. Then, the distance between the edges of the scratch was calculated in the same field at indicated times. Three repeated experiments were carried out.

\section{Transwell assay}

Transwell assay was conducted to investigate the invasion of CAL27 and TCA8113 cells. $3 \times 10^{5}$ cells were seeded into the upper chambers with $200 \mu \mathrm{L}$ serum-free cell culture medium and complete cell culture medium was added into the low chambers. The cells were cultured at $37^{\circ} \mathrm{C}$ with $5 \%$ $\mathrm{CO}_{2}$ for $24 \mathrm{~h}$ and fixed by $4 \%$ paraformaldehyde for $15 \mathrm{~min}$ and stained with $0.1 \%$ crystal violet. The cells number was observed in 5 random fields under a microscope. Three repeated experiments were carried out.

\section{Xenograft study}

The animal experiments protocol was approved by the committee of the ethics of animal experiments of female 5 -week-old BALB/c nude mice were purchased from the Hunan SJA Laboratory Animal Co., Ltd., Hunan, China $5 \times$ $10^{6}$ CAL27 cells transfected with KIF18A shRNA or control plasmids were inoculated into the upper back of mice. Tumor growth was monitored every 7 days and the tumor volumes were calculated. After 7 weeks, tumors were harvested and mice were sacrificed. The tumor tissues were used for subsequent experiments. Moreover, to study the connection between KIF18A and metastasis in vivo, $5 \times 10^{4}$ CAL27 cells transfected with KIF18A shRNA or control shRNA plasmids were inoculated into tail vein of mice. Lung metastatic tumors were harvested followed the above protocol.

\section{Statistical analysis}

All the data were analyzed by SPSS version 23.0. The quantitative data were assessed as mean \pm SEM, and Student's $t$-test was used to analyze the two groups. The chisquare test was used to compare the counting data. KaplanMeier method was used to evaluate the patients' prognosis. $P<0.05$ was considered to be significant.

\section{Results}

High KIF18A expression associated with clinical-pathological features of OTSCC patients

To investigate the connections between KIF18A expression and clinical-pathological features of OTSCC patients, we performed immunohistochemistry staining of 82 patients diagnosed with OTSCC. Staining of patient tumor tissues showed high and low cytoplasmic positivity staining in OTSCC cells (Figs. 1A and 1B). Based on the staining results, they were divided into two groups: high KIF18A expression group and low expression group. We demonstrated that KIF18A expression was significantly associated with the lymph node metastasis and clinical stages (Phases I-II, III-IV) of OTSCC patients $(P<0.05)$. However, no significant connections between KIF18A and ages, genders, tumor stage, and tumor differentiation (high and low differentiation) were found (Tab. 1).

High KIF18A expression associated with the prognosis of OTSCC patients

To further investigate the clinical significance of KIF18A, overall survival (OS) and progression-free survival (PFS) was generated based on Kaplan-Meier method. The postoperative follow-up method was: every three months in the first year and then every six months in the later years. The results showed that KIF18A was significantly related to the prognosis of patients (Fig. 1C). Patients with high KIF18A expression tended to gain short OS and PFS $(P<0.05)$.

Construction of stable KIF18A knockdown OTSCC cell lines Due to the increased KIF18A expression in OTSCC patients, short hairpin RNAs targeting KIF18A was stably transduced in OTSCC cell lines, CAL27 and TCA8113, to perform the loss-of-function study. The knockdown efficiency in control and KIF18A shRNA groups was verified by RT-qPCR and immunoblotting. The results showed that KIF18A mRNA and protein levels were dramatically decreased in both cell lines compared to control $(P<0.05$; Fig. 2$)$. Thus, we have effectively generated KIF18A-depleted OTSCC cell lines.

\section{KIF18A knockdown inhibited OTSCC cells proliferation,} migration, and invasion in vitro

To explore the effect of KIF18A on tumor cell proliferation, we performed colony formation and MTT assays in CAL27 and TCA8113 cell lines. As was expected, silencing of KIF18A expression could inhibit the proliferation in both cell lines compared with control $(P<0.05$; Figs. $3 \mathrm{~A}$ and $3 \mathrm{~B})$. Moreover, we detected some important proliferation-related proteins to further investigate the related mechanism of KIF18A. The protein level of Ki67 and PCNA was showed to be dramatically decreased in KIF18A silencing cell lines 

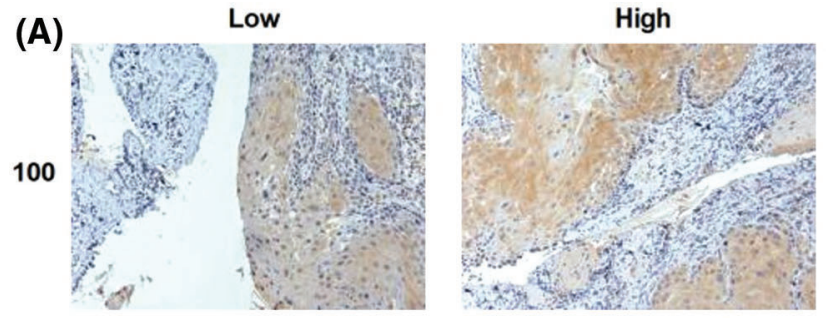

(B)
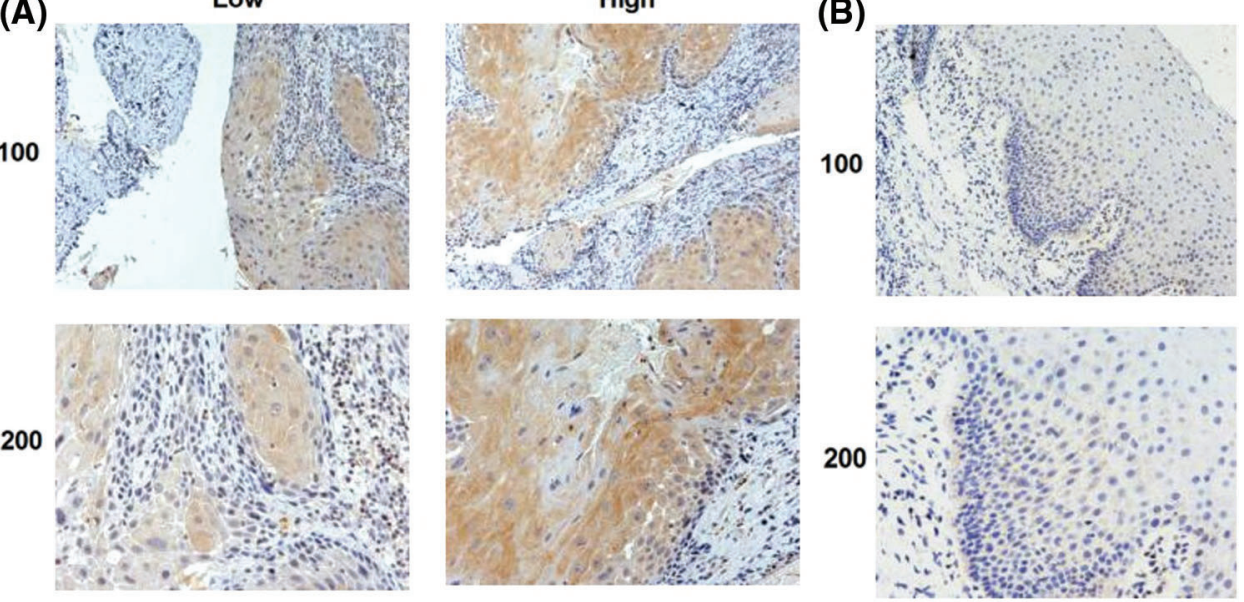

(C)
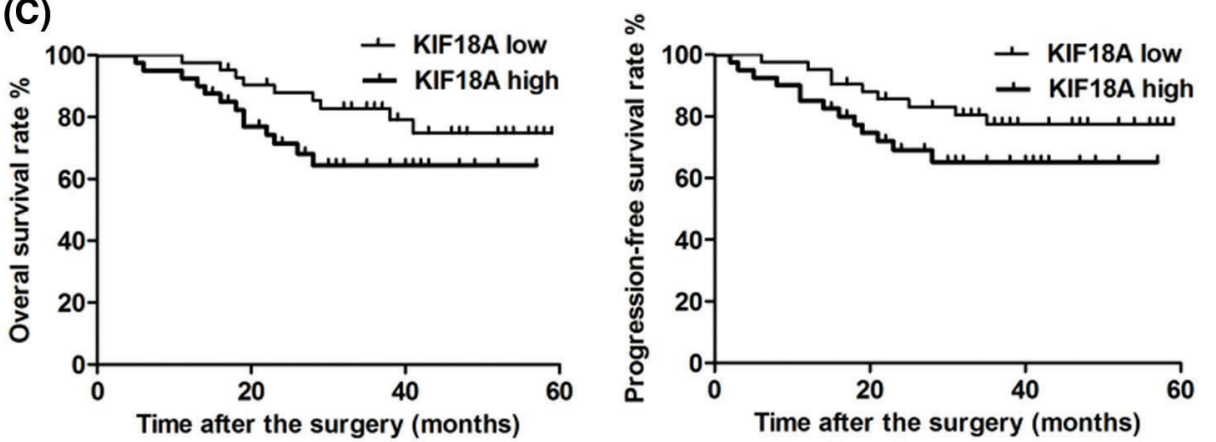

FIGURE 1. KIF18A protein expression level associated with prognosis OTSCC patients. (A) Low and high KIF18A protein expression in OTSCC patients by immunohistochemistry were shown. (B) The KIF18A protein expression in the tumor-adjacent tissues. (C) The connections between KIF18A and the overall (OS) and progress-free (PFS) survival rates of patients were generated by Kaplan-Meier method, and we found high KIF18A protein expression was related to short OS and PFS in the patients of OTSCC.
TABLE 1

Relationships of KIF18A and clinicopathological characteristics in 82 patients with tongue squamous cell carcinoma

\begin{tabular}{|c|c|c|c|c|c|}
\hline \multirow{3}{*}{ Feature } & \multirow{3}{*}{$\begin{array}{l}\text { All } \\
\mathrm{N}=82\end{array}$} & \multicolumn{2}{|c|}{ KIF18A expression } & \multirow[t]{3}{*}{$x^{2}$} & \multirow[t]{3}{*}{$P$} \\
\hline & & Low & High & & \\
\hline & & $\mathrm{N}=42$ & $\mathrm{~N}=\mathbf{4 0}$ & & \\
\hline Age (year) & & & & 0.532 & 0.466 \\
\hline$<65$ & 50 & 24 & 26 & & \\
\hline$\geq 65$ & 32 & 18 & 14 & & \\
\hline Gender & & & & 1.717 & 0.190 \\
\hline Male & 45 & 26 & 19 & & \\
\hline Female & 37 & 16 & 21 & & \\
\hline Tumor stage & & & & 2.513 & 0.113 \\
\hline $\mathrm{T}_{1-2}$ & 36 & 22 & 14 & & \\
\hline $\mathrm{T}_{3-4}$ & 46 & 20 & 26 & & \\
\hline Differentiation & & & & 3.241 & 0.072 \\
\hline Low & 24 & 16 & 8 & & \\
\hline High & 58 & 26 & 32 & & \\
\hline Clinical stage & & & & 4.228 & $0.040^{*}$ \\
\hline I-II & 34 & 22 & 12 & & \\
\hline III-IV & 48 & 20 & 28 & & \\
\hline $\begin{array}{l}\text { Lymph node } \\
\text { metastasis }\end{array}$ & & & & 5.859 & $0.015^{*}$ \\
\hline Yes & 38 & 14 & 24 & & \\
\hline No & 44 & 28 & 16 & & \\
\hline
\end{tabular}

$(P<0.05$; Figs. 3C and 3D). Taken together, our results showed that through regulating Ki67 and PCNA expression, depletion of KIF18A protein could reduce the proliferation ability of OTSCC cells.

In addition, to explore the ability of KIF18A in migration and invasion of tumor cells, wound healing and Transwell assays were performed. Depletion of KIF18A in OTSCC cells, the ability of migration and invasion were also reduced $(P<$ 0.05; Figs. $4 \mathrm{~A}$ and $4 \mathrm{~B})$. We checked several key proteins that may reflect the migration and invasion ability in tumor cells to investigate the related mechanism. The results showed that the protein expression level of matrix metalloproteinases-7 (MMP7) and MMP9, which were associated with the movement of tumor cells, was dramatically decreased by silencing of KIF18A in tumor cells $(P<0.05$; Figs. $4 \mathrm{C}$ and $4 \mathrm{D})$. In conclusion, we found that KIF18A might participate in the migration and invasion of tumor cells through the regulation of MMP7 and MMP9.

\section{Knockdown KIF18A restricts the proliferation and metastasis} of tumors in vivo

We further examined the biological roles of KIF18A in OTSCC tumorigenesis by xenograft study in mice. CAL27 cells transfected with KIF18A shRNA or control shRNA were inoculated subcutaneously into the upper back of nude mice and mice tail vein. Tumor volume were calculated every 7 days to generate the growth curve. After 7 weeks, tumors were harvested and mice were sacrificed. The results showed that tumor growth in the KIF18A shRNA group was slower than that of the control $(P<0.05$; Figs. 5A and $5 \mathrm{~B})$. To validate the expression of KIF18A in mice tumors, 
(A)

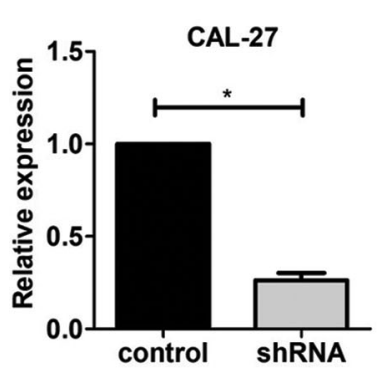

(B)

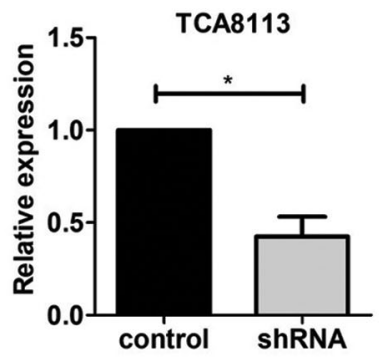

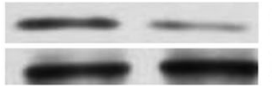

KIF18A $\beta$-actin

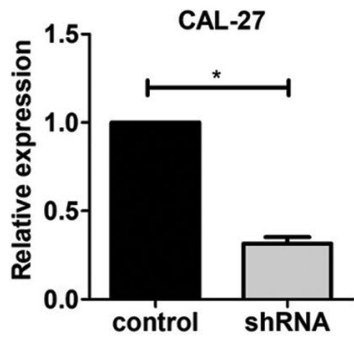

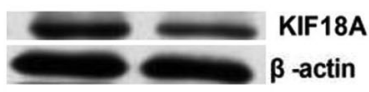

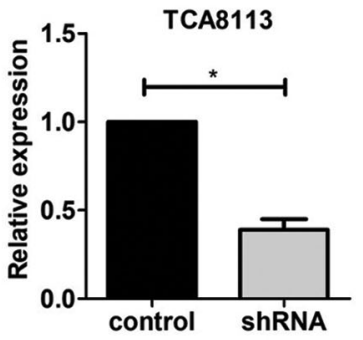

FIGURE 2. Construction of stable KIF18A knockdown OTSCC cell lines. (A) and (B) RT-qPCR and immunoblotting. KIF18A mRNA and protein levels of KIF18A in CAL27 and TCA8113 cell lines were significantly decreased in KIF18A shRNA group compared to control $\left(\mathrm{N}=3,{ }^{*} P<0.05\right)$.

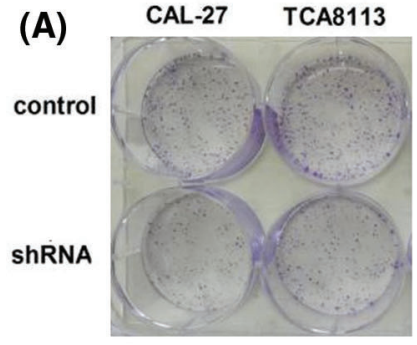

(C)

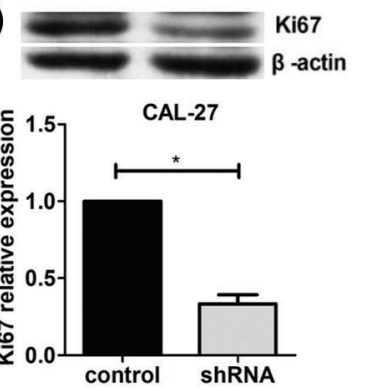

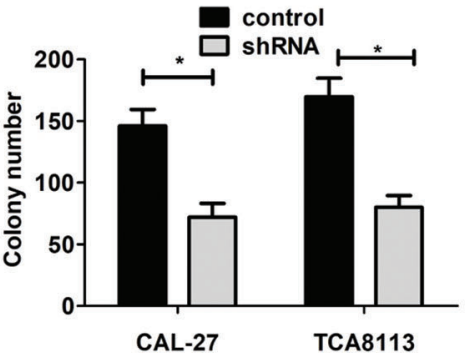
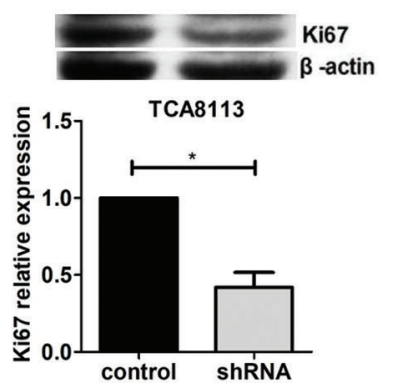

(B)
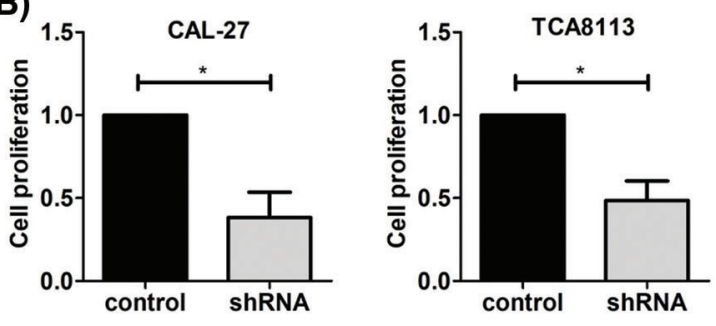

(D)
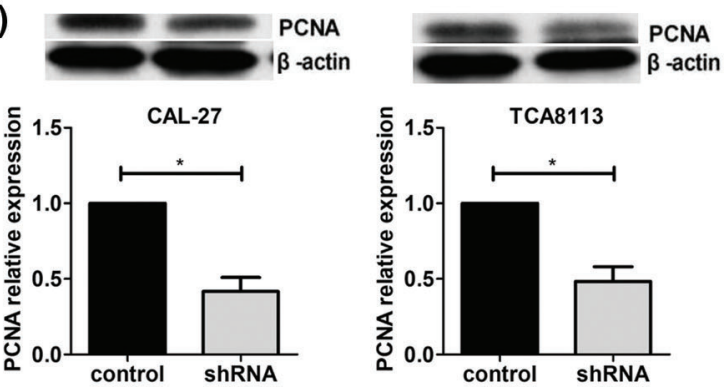

FIGURE 3. KIF18A knockdown inhibited OTSCC cells proliferation through Ki67 and PCNA regulation. (A) Colony formation assay. The results demonstrated that in CAL27 and TCA8113 cell lines, colonies in KIF18A shRNA group were significantly less than control $\left(\mathrm{N}=3,{ }^{*} P<0.05\right)$. (B) MTT assay. The results showed that OD values in KIF18A shRNA group were lower than control $\left(\mathrm{N}=3,{ }^{*} P<0.05\right)$. (C-D) Immunoblotting. The results showed that the Ki67 and PCNA proteins were significantly downregulated by silencing of KIF18A $\left(\mathrm{N}=3,{ }^{\star} P<0.05\right)$.

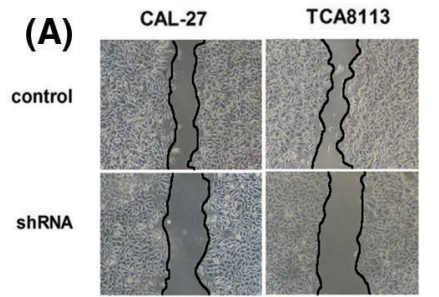

(C)

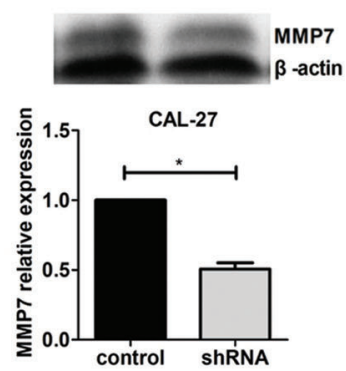

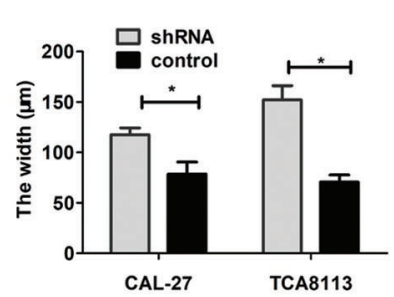
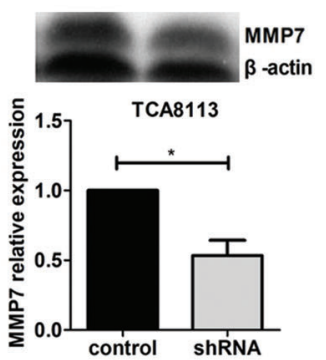
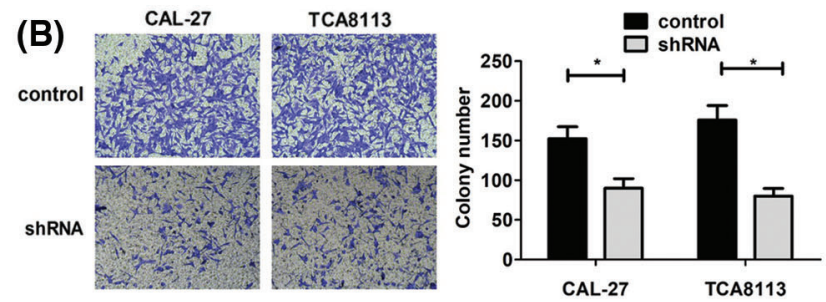

(D)
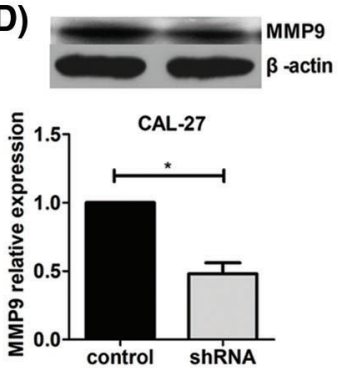
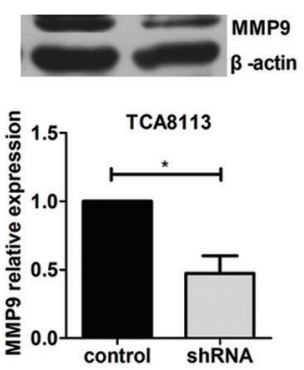

FIGURE 4. KIF18A knockdown inhibited the migration and invasion of OTSCC cells by regulating MMP7 and MMP9. (A) Wound healing assay. The scratch gap in KIF18A shRNA group were wider than control groups, respectively $\left(\mathrm{N}=3,{ }^{*} P<0.05\right)$. (B) Transwell assay. The number of invasive cells in KIF18A shRNA groups was significantly decreased than control ( $\left.\mathrm{N}=3,{ }^{*} P<0.05\right)$. (C-D) Immunoblotting. The results showed that the expression of MMP7 and MMP9 proteins was lower in KIF18A shRNA group than control $\left(\mathrm{N}=3,{ }^{*} P<0.05\right)$. 
(A)
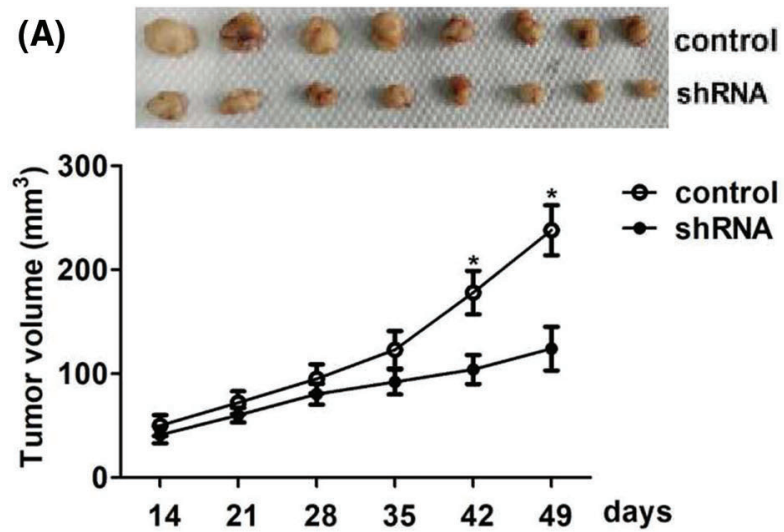

(B)
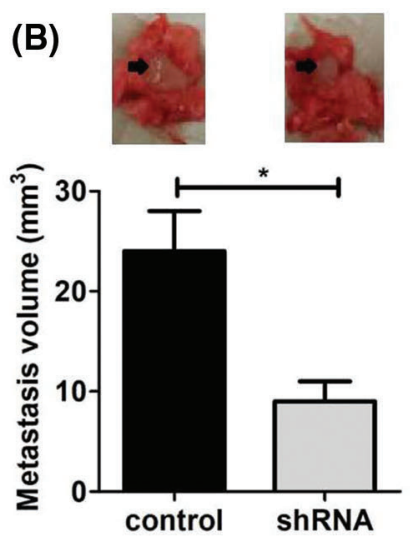

(C)

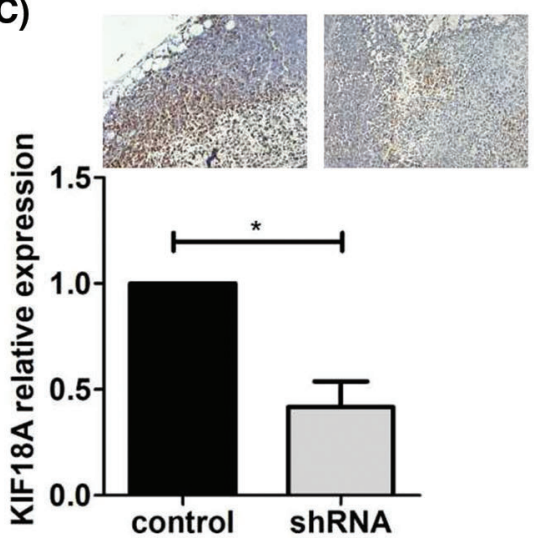

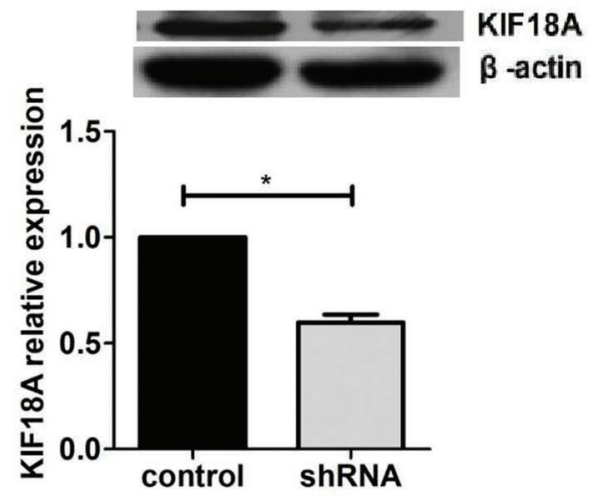

FIGURE 5. The role of KIF18A in tumor growth and metastasis in vivo. (A) and (B) Xenograft assay. The tumor growth in KIF18A shRNA group was significantly slower than control and the tumor volume of KIF18A shRNA group was smaller than control $\left(\mathrm{N}=8,{ }^{*} \mathrm{P}<\right.$ 0.05). (C) Immunohistochemistry and Immunoblotting. The results showed that KIF18A protein level was significantly decreased in KIF18A shRNA group $\left(\mathrm{N}=8,{ }^{\star} P<0.05\right)$. Immunohistochemistry $\times 100$. immunoblotting and immunohistochemistry (IHC) analysis were performed. The results revealed that KIF18A protein level was significantly decreased in KIF18A shRNA transfection group $(P<0.05$; Fig. 5C). Taken together, we demonstrated that the depletion of KIF18A may reduce the growth and metastasis of tumors in vivo.

\section{Discussion}

Tongue cancer is a common malignant tumor of oral and maxillofacial region (Ferlay et al., 2015; Li et al., 2013; Patel et al., 2011; Anaya-Saavedra et al., 2008). Most tongue cancers are OTSCC, and OTSCC has a high degree of malignancy and mortality (Elango et al., 2007; Pintos et al., 2008; Chiodo et al., 1986). Targeted therapy shows a good therapeutic prospect (Ribeiro et al., 2016; Arteaga and Baselga, 2012). The effective target treatment for OTSCC is urgently needed to improve the prognosis. A previous study underlines as D-dSCs could represent a useful steroidogenic model for the development of the target and gene therapies in OSCC. They showed that proliferation was promoted by estradiol. Moreover, no significant difference in cell death levels was detected among treatments. Therefore, estradiol could serve as a promising therapeutic drug (Boccellino et al., 2019). Interestingly, we found a protein which belonged to the kinesin family, KIF18A, was highly expressed in the tissues of OTSCC. KIF18A promotes OTSCC development and might serve as a novel therapeutic target for the treatment of OTSCC.

Recently, it has been reported that KIF18A dysregulation is functionally significant in several types of cancers
(Kasahara et al., 2016; Zhang et al., 2010; Nagahara et al., 2011; Liao et al., 2014; Chen and Shang, 2017). Chen et al. (2017) showed that the abnormal expression of kinesins was involved in the progression and prognosis of liver cancer from a retrospective study of 295 patients. Luo et al. (2018) further demonstrated that in two types of HCC invasive cells lines, depletion of KIF18A reduced the cell proliferation, cell invasion, and migration. Previous studies have shown that the regulation of early mitosis in the tumor might lead to synovial sarcoma, suggesting that abnormal expression of KIF18A in cancer may lead to dysregulation of cell mitosis control (Przybyl et al., 2014). Zhang et al. (2010) reported that overexpression of KIF18A was significantly associated with breast cancer in advanced grade, metastasis, and prognosis. Stably ectopic overexpression of KIF18A in MCF-7 cells caused multinuclear formation which was associated with tumor carcinogenesis. Meanwhile, overexpression of KIF18A could interfere with the cytoskeletal microtubule array dynamics and the formation of mitotic spindles, eventually leading to cell transformation, tumor development, and metastasis (Mayr et al., 2007). Ablation of KIF18A expression significantly inhibited the proliferation of tumor cells in vitro and in vivo. In addition, KIF18A knockdown significantly inhibited tumor growth and metastasis in breast cancer and HCC (Luo et al., 2018). All of the above studies have suggested that KIF18A may serve as a novel biomarker of tumors.

In this study, we investigated both the clinical features and biological function of KIF18A in OTSCC carcinogenesis. By immunohistochemistry analysis, we found that elevated KIF18A expression was related to the tumor differentiation, 
lymph node metastasis, and clinical stages of OTSCC patients. Previous studies showed that due to rapidly localized invasion and early metastasis to regional lymph nodes, effective treatment approaches should focus on how to suppress OTSCC invasion and migration (Sciubba, 2001). Based on the high expression level of KIF18A in OTSCC patients, we used shRNA-mediated assay to knock down KIF18A in OTSCC cell lines to further investigate its biological activities in OTSCC cells. The results showed that KIF18A knockdown significantly inhibited tumor proliferation, migration, and invasion in vitro. These observations were consistent with those with high levels of KIF18A expression showing invasive prognosis. Scales et al. (2017) showed that KIF18A might lead to abnormality of cell mitosis control and promote cell division, however, the related mechanism was still not clear. To better investigate the mechanisms of KIF18A in OTSCC cell lines, we checked several key proteins that were involved in cancer-related functions. Our results showed that silencing of KIF18A could dramatically decrease the protein expression level of Ki67, PCNA, which promoted cell proliferation. Besides, we also demonstrated that through the MMP-7/MMP-9-related pathway, KIF18A promoted OTSCC cancer cell invasion and metastasis in vitro. These findings coincided with some related studies in the KIF18A research (Stumpff et al., 2008; Mayr et al., 2007; Luo et al., 2018). We generated KIF18A stabledepleted OTSCC cell lines and inoculated subcutaneously and injected into the tail vein of nude mice. The results revealed that depletion of KIF18A may decrease the growth and metastasis of tumors, and high expression of KIF18A may be involved in the tumor formation in vivo. Moreover, kinesin motor proteins have been considered as the effective targets for the anticancer treatment (Honore et al., 2005; Marcus et al., 2005; Bhat and Setaluri, 2007; Huszar et al., 2009). BTB1, the first small molecule inhibitor of KIF18A, reversibly inhibited the ATPase activity of the recombinant motor domain of KIF18A in vitro (Catarinella et al., 2009). Thus, KIF18A could serve as an invaluable probe in the treatment of OTSCC. For OSTCC patients, further research on KIF18A is still needed to elucidate its exact mechanisms.

As we know, KIFs were involved in the progression of multiple types of cancers such as bladder cancer and breast cancer (Kasahara et al., 2016; Stumpff et al., 2008). Herein, we identified another kinesin, KIF18A, was a potential prognostic indicator of OTSCC. Our data further confirmed the impairment of cell proliferation and invasion of this type of cancer caused by KIF18A depletion. Our results, together with previous studies, indicated that KIFs played critical role in cancer development, and provided novel therapeutic targets for the treatment of OTSCC. Notably, several studies demonstrate the function of KIFs on cellular processes including cytoskeleton dynamic and organization, spindle formation and chromosome segregation (Stumpff et al., 2008). Therefore KIFs might affect cell proliferation and migration of various cancers, including OTSCC. However, the precise molecular mechanism underlying KIF18A promoting the progression of OTSCC needs further study. The single center study with small number of cases and the short follow up are the limitation in this study. In addition, the lack of univariate and multivariate survival analysis is another important limitation of study.
In conclusion, our results showed that KIF18A expression was significantly associated with the progression of OSTCC and poor clinical outcomes. Depletion of KIF18A could decrease the proliferation, migration, and invasion of cancer cells in vitro. In addition, KIF18A depletion reduced the tumor growth and metastasis in vivo. Our results therefore showed that KIF18A might serve as a biomarker for OSTCC and a target for therapeutic intervention in the future.

Availability of Data and Materials: The datasets used and/or analyzed during the current study are available from the corresponding author on reasonable request.

Author Contribution: XL, XY, and CP designed this research; $\mathrm{XL}, \mathrm{XY}$, and JX performed experiments; XL, XY, and $\mathrm{CP}$ contributed to the writing of the manuscript; $\mathrm{MW}$ analyzed all the immunohistochemistry results. XL and XY collected clinical specimens; CP analyzed the data.

Ethics Statement: This study was conducted ethically in accordance with the World Medical Association Declaration of Helsinki and approved by the Ethics Committee of the Second Hospital of Tianjin Medical University. All patients enrolled in the study have signed informed consent.

Funding Statement: The authors received no specific funding for this study.

Conflicts of Interest: The authors declare that they have no conflicts of interest to report regarding the present study.

\section{References}

Anaya-Saavedra G, Ramírez-Amador V, Irigoyen-Camacho ME, García-Cuellar CM, Guido-Jiménez M et al. (2008). High association of human papillomavirus infection with oral cancer: A case-control study. Archives of Medical Research 39: 189-197. DOI 10.1016/j.arcmed.2007.08.003.

Arteaga CL, Baselga J (2012). Impact of genomics on personalized cancer medicine. Clinical Cancer Research 18: 612-618. DOI 10.1158/1078-0432.CCR-11-2019.

Bhat K, Setaluri V (2007). Microtubule-associated proteins as targets in cancer chemotherapy. Clinical Cancer Research 13: 2849. DOI 10.1158/1078-0432.CCR-06-3040.

Boccellino M, di Stasio D, Dipalma G, Cantore S, Ambrosio P et al. (2019). Steroids and growth factors in oral squamous cell carcinoma: Useful source of dental-derived stem cells to develop a steroidogenic model in new clinical strategies. European Review for Medical and Pharmacological Sciences 23: 8730-8740. DOI 10.26355/eurrev_201910_19267.

Braun J, Möckel MM, Strittmatter T, Marx A, Groth U, Mayer TU (2015). Synthesis and biological evaluation of optimized inhibitors of the mitotic kinesin Kif18A. ACS Chemical Biology 10: 554. DOI 10.1021/cb500789h.

Catarinella M, Grüner T, Strittmatter T, Marx A'Mayer TU (2009). BTB-1: A small molecule inhibitor of the mitotic motor protein Kif18A. Angewandte Chemie International Edition 48: 9072-9076. DOI 10.1002/anie.200904510.

Chen GY, Shang JJ (2017). Expression and clinical significance of KIF18A protein in gastric cancer tissues. PLA Medical Journal 29: 47-50. DOI 10.1016/j.prp.2017.09.009.

Chen J, Shu L, Shu Z, Li G (2017). Kinesin superfamily protein expression and its association with progression and 
prognosis in hepatocellular carcinoma. Journal of Cancer Research and Therapeutics 13: 651. DOI 10.4103/jcrt.JCRT_491_17.

Chen QI, Cao B, Nan N, Wang YU, Zhai XU et al. (2016). Elevated expression of KIF18A enhances cell proliferation and predicts poor survival in human clear cell renal carcinoma. Experimental \& Therapeutic Medicine 12: 377-383. DOI 10.3892/etm.2016.3335.

Chiodo GT, Eigner T, Rosenstein DI (1986). Oral cancer detection. The importance of routine screening for prolongation of survival. Postgraduate Medicine 80: 231. DOI 10.1007/ BF01712057.

Elango KJ, Suresh A, Erode EM, Subhadradevi L'Kuriakose MA (2007). Role of human papilloma virus in oral tongue squamous cell carcinoma. Asian Pacific Journal of Cancer Prevention 12: 889-896. DOI 10.1097/01.cad.0000390767.85658.83.

Ferlay J, Soerjomataram I, Dikshit R, Eser S, Mathers C et al. (2015). Cancer incidence and mortality worldwide: Sources, methods and major patterns in GLOBOCAN 2012. International Journal of Cancer 136: E359-E386. DOI 10.1002/ijc.29210.

Frisch SM, Screaton RA (2001). Anoikis mechanism. Current Opinion in Cell Biology 13: 555-562. DOI 10.1016/S0955-0674(00)00251-9.

Honore S, Pasquier E, Braguer D (2005). Understanding microtubule dynamics for improved cancer therapy. Cellular \& Molecular Life Sciences 62: 3039-3056. DOI 10.18632/oncotarget.15874.

Huszar D, Theoclitou ME, Skolnik J, Herbst R (2009). Kinesin motor proteins as targets for cancer therapy. Cancer \& Metastasis Reviews 28: 197-208. DOI 10.1007/s10555-009-9185-8.

Kasahara M, Nagahara M, Nakagawa T, Ishikawa T, Sato T et al. (2016). Clinicopathological relevance of kinesin family member 18A expression in invasive breast cancer. Oncology Letters 12: 1909-1914. DOI 10.3892/ol.2016.4823.

Li R, Koch WM, Fakhry C, Gourin CG (2013). Distinct epidemiologic characteristics of oral tongue cancer patients. OtolaryngologyHead and Neck Surgery 148: 792-796. DOI 10.1177/ 0194599813477992.

Liao W, Huang G, Liao Y, Yang J, Chen Q et al. (2014). High KIF18A expression correlates with unfavorable prognosis in primary hepatocellular carcinoma. Oncotarget 5: 10271. DOI 10.18632/oncotarget.2082.

Luo W, Liao M, Liao Y, Chen X, Huang C et al. (2018). The role of kinesin KIF18A in the invasion and metastasis of hepatocellular carcinoma. World Journal of Surgical Oncology 16: 36. DOI 10.1186/s12957-018-1342-5.

Marcus AI, Peters U, Thomas SL, Garrett S, Zelnak A et al. (2005). Mitotic kinesin inhibitors induce mitotic arrest and cell death in taxol-resistant and -sensitive cancer cells. Journal of Biological Chemistry 280: 11569-11577. DOI 10.1074/jbc. M413471200.

Mayr MI, Hümmer S, Bormann J, Grüner T, Adio S et al. (2007). The human kinesin Kif18A is a motile microtubule depolymerase essential for chromosome congression. Current Biology 17: 488-498. DOI 10.1016/j.cub.2007.02.036.

Nagahara M, Nishida N, Iwatsuki M, Ishimaru S, Mimori K et al. (2011). Kinesin 18A expression: Clinical relevance to colorectal cancer progression. International Journal of Cancer 129: 2543-2552. DOI 10.1002/ijc.25916.

Patel SC, Carpenter WR, Tyree S, Couch ME, Weissler M et al. (2011). Increasing incidence of oral tongue squamous cell carcinoma in young white women, age 18 to 44 years. Journal of Clinical Oncology 29: 1488-1494. DOI 10.1200/ JCO.2010.31.7883.

Pintos J, Black MJ, Sadeghi N, Ghadirian P, Zeitouni AG et al. (2008). Human papillomavirus infection and oral cancer: A casecontrol study in Montreal. Canada Oral Oncology 44: 242250. DOI 10.1016/j.oraloncology.2007.02.005.

Przybyl J, Sciot R, Wozniak A, Schöffski P, Vanspauwen V et al. (2014). Metastatic potential is determined early in synovial sarcoma development and reflected by tumor molecular features. International Journal of Biochemistry \& Cell Biology 53: 505-513. DOI 10.1016/j.biocel.2014.05.006.

Ribeiro IP, Barroso L, Marques F, Melo JB, Carreira IM (2016). Early detection and personalized treatment in oral cancer: The impact of omics approaches. Molecular Cytogenetics 9: 85. DOI 10.1186/s13039-016-0293-1.

Scales M, Chubb D, Dobbins SE, Johnson DC, Li N et al. (2017). Search for rare protein altering variants influencing susceptibility to multiple myeloma. Oncotarget 8: 3620336210. DOI 10.18632/oncotarget.15874.

Sciubba JJ (2001). Oral cancer. The importance of early diagnosis and treatment. American Journal of Clinical Dermatology 2: 239251. DOI 10.2165/00128071-200102040-00005.

Stumpff J, von Dassow G, Wagenbach M, Asbury C, Wordeman L (2008). The kinesin-8 motor Kif18A suppresses kinetochore movements to control mitotic chromosome alignment. Developmental Cell 14: 252-262. DOI 10.1016/j.devcel.2007.11.014.

Zhang C, Zhu C, Chen H, Li L, Guo L et al. (2010). Kif18A is involved in human breast carcinogenesis. Carcinogenesis 31: 16761684. DOI 10.1093/carcin/bgq134.

Zhu C (2005). Functional analysis of human microtubule-based motor proteins, the kinesins and dyneins, in mitosis/ cytokinesis using RNA interference. Molecular Biology of the Cell 16: 3187-3199. DOI 10.1091/mbc.E05-02-0167. 